Revista de Economia Política, vol. 29, $n^{\circ} 3$ (115), pp. 173-190, julho-setembro/2009

\title{
A tendência à harmonização internacional da proteção de patentes e seus problemas
}

RONALDO FIANI*

The trend towards international harmonization of patents' protection and its
problems. TRIPS agreement has been a milestone in the present trend towards inter-
national harmonization of intellectual property's protection (including patents' pro-
tection), where harmonization means to increase the protection level in developing
countries according to the more rigorous standards of developed ones. Considering
the economic theory of patents together with historical and empirical evidences, it
is argued that international harmonization of patents' protection as it is settled by
TRIPS agreement is not favorable to developing countries' economic development.
Keywords: patents; intellectual property rights; developing countries; TRIPS.
JEL Classification: P14; O34; F13.

\section{INTRODUÇÃO}

Desde o início da última década do século passado verifica-se uma tendência crescente à uniformização internacional da proteção de direitos de propriedade em geral, e de patentes em particular, aos mesmos níveis elevados de proteção oferecida nos países desenvolvidos. O acordo TRIPS (Agreement on Trade-Related Aspects of Intellectual Property Rights), obtido no final da rodada Uruguai em 1994, consolidou essa tendência.

Ao aumentar a proteção a patentes, a tendência de harmonização da proteção de direitos de propriedade intelectual pode encarecer e limitar o acesso à tecnologia internacional. Justifica-se, assim, investigar se a harmonização internacional da proteção de direitos de propriedade intelectual favorece o desenvolvimento econômico.

\footnotetext{
* Professor Adjunto e Diretor do Núcleo de Estudos Internacionais da Universidade Federal do Rio de Janeiro. E-mail: rfiani@gmail.com. Submetido: Novembro 2007; Aprovado: Março 2008.
} 
Com esse objetivo, este artigo se organiza em cinco seções. Na primeira seção é apresentada a controvérsia em torno dos efeitos de uma proteção mais severa de patentes sobre a inovação, em especial no ritmo do progresso tecnológico de países em desenvolvimento. A partir daí a seção seguinte discute a relação entre proteção de patentes e desenvolvimento econômico.

A terceira seção apresenta o acordo TRIPS e de que forma ele busca implementar a harmonização internacional da proteção de patentes. A quarta seção discute a importância relativa do comércio internacional e do investimento direto estrangeiro na difusão de tecnologia, concluindo que o investimento direto estrangeiro deve ser o canal mais importante de difusão tecnológica e indagando acerca dos efeitos da proteção de patentes para a atração deste investimento. Uma última seção de conclusões resume os resultados obtidos.

\section{A CONTROVÉRSIA ECONÔMICA SOBRE PATENTES}

É reconhecido que, caso os rendimentos de uma inovação, seja de produto ou de processo, não possam ser apropriados pelo inventor, ou seja, se a inovação puder ser reproduzida por um imitador a um custo inferior ao do inovador, este último não terá incentivos para realizar todos os gastos necessários ao desenvolvimento da inovação. A questão é que, embora as patentes sejam um dos instrumentos de apropriação de rendimentos de inovações, as patentes não constituem a única forma possível de apropriação desses rendimentos (segredos comerciais são outra forma), e elas possuem um custo para a sociedade.

Com relação à apropriação dos rendimentos das inovações, é preciso inicialmente considerar o fato de que a proteção oferecida pelas patentes não possui a mesma importância para todos os setores da indústria. Com efeito, em determinados setores a engenharia reversa não parece ser capaz de fornecer as informações necessárias para a apropriação da inovação por outros agentes. Esse ponto muitas vezes é ofuscado pela adoção da hipótese implícita de que as patentes são sempre a melhor forma de proteger os direitos sobre uma inovação.

De fato, como explicam Scherer (1984) e Scherer e Ross (1990), outros fatores podem permitir ao inovador garantir a apropriação dos ganhos extraordinários da inovação sem o recurso ao patenteamento. Em primeiro lugar, caso se verifique no setor em questão uma curva de aprendizado longa e com inclinação significativa, as vantagens de ser o primeiro a mover podem ser tão elevadas a ponto de dispensar a proteção formal oferecida pelas patentes.

Em segundo lugar, o custo de imitar uma inovação bem-sucedida dificilmente é nulo. Frequentemente, uma parte dos gastos do inovador original em pesquisa e desenvolvimento tem de ser reinvestida, gerando um custo para aquele que tenta reproduzir a inovação bem-sucedida. Esse custo é o custo de absorver nova tecnologia, e ele pode retardar bastante a difusão da inovação, permitindo ao inovador original se apropriar de ganhos elevados nesse intervalo. Por último, também pode haver um efeito-reputação, por ter sido o primeiro a realizar a inovação. 
Por exemplo, Ordover (1991) apresenta uma visão que difere da teoria convencional. Ordover irá afirmar, entre outros argumentos, que uma proteção "fraca" de patentes não precisa ser, necessariamente, um fator contrário ao crescimento econômico. O ponto de partida de Ordover é o fato de que, afinal de contas, a proteção das patentes não é tão excludente assim, pois o titular de uma patente tem de revelar o conteúdo da patente publicamente. Isso, obviamente, inclui revelar esse conteúdo aos seus rivais efetivos ou potenciais. Essa revelação (disclosure) pode afetar adversamente a vantagem que a patente confere ${ }^{1}$.

Para evitar que a revelação ameace seus interesses, o inovador pode optar por proteger a patente como um segredo comercial (trade secret). Ordover (1991) considera esta estratégia especialmente interessante em processos industriais para os quais a engenharia reversa não pode ser facilmente aplicada.

Há pouco publicado na literatura econômica sobre segredos comerciais. Um dos poucos trabalhos a tratar especificamente do tema com aportes originais é Friedman, Landes e Posner (1991). Esses autores indagam as razões de uma empresa escolher não patentear uma informação, mantendo-a como segredo comercial e abrindo mão da proteção que as patentes oferecem, ou seja, sob que condições as patentes podem (ou não) oferecer proteção efetiva aos seus proprietários. Friedman, Landes e Posner (1991) consideram três situações possíveis, em que o segredo comercial se mostra mais interessante do que patentes, do ponto de vista do inovador:

1. inovador possui conhecimento patenteável, e acredita que vai levar um tempo tão longo, ou quase tão longo quanto a duração da patente, para que algo igual seja inventado, mas a inovação possui valor comercial modesto.

2. inovador possui conhecimento patenteável e acha que vai levar mais tempo do que a duração da patente para que algo igual seja inventado.

3. inovador possui um novo conhecimento que não é patenteável, mas acredita que vai levar tempo suficiente para que a ideia seja reproduzida, de tal forma que ele pode obter um retorno considerável.

Assim, haveria pelo menos três situações em que segredos comerciais podem ser mais interessantes para o inovador do que a proteção oferecida pelas patentes. Mas cumpre também indagar acerca das evidências empíricas em relação ao valor da proteção oferecida pelas patentes aos inovadores.

A importância das patentes para a inovação foi objeto de pesquisa empírica desde os anos oitenta do século passado. Mansfield (1986), estudando uma amostra aleatória de 100 empresas de 12 indústrias diferentes, no período 1981-1983, verificou que em metais primários, equipamento elétrico, instrumentos, equipa-

\footnotetext{
${ }^{1}$ Contudo, a possibilidade de que a revelação do conteúdo da patente diminua a vantagem do inovador é polêmica. James Bessen (2005) argumenta que as firmas que efetivamente consultam patentes visam primordialmente a: manter-se atualizadas quanto às estratégias de seus competidores ou analisar possíveis infrações de suas próprias patentes.
} 
mento de escritório, motores para veículos, borracha e têxteis eram poucas as invenções comercializadas que poderiam ser explicadas pela proteção oferecida pelas patentes. Já no setor farmacêutico e químico, as patentes produziam um forte efeito positivo na introdução das inovações.

Da mesma forma, Mansfield, Schwartz e Wagner (1981) verificaram que a importância da proteção oferecida pelas patentes é significativamente maior para a indústria farmacêutica do que para as demais indústrias, como a indústria eletrônica. Como explicam Mansfield, Schwartz e Wagner (1981, p. 913), dado que frequentemente não é possível reproduzir o processo apenas a partir da engenharia reversa do produto, isso reduz o interesse na proteção das inovações de processo por patentes (ver também Levin et al., 1987).

Desse modo, alternativas às patentes para a apropriação das rendas de inovações de processos podem se mostrar muito mais interessantes, tais como vantagens de ser o pioneiro (lead time), vantagens de aprender fazendo e o simples segredo comercial. Há evidências de que "Secrecy, though not considered as effective as lead time and learning advantages, was still considered more effective than patents in protecting processes (Levin et al., 1987, p. 795).

$\mathrm{Na}$ verdade, a crítica de que nem tudo que pode ser patenteado é necessariamente protegido de forma mais eficiente pela patente não é recente. Como explicam Machlup e Penrose (1950, pp. 26-27), entre as várias críticas desenvolvidas no século XIX ao sistema de patentes, em particular à justificativa das patentes pelo seu incentivo à revelação da descoberta, já se encontrava o argumento de que, na verdade, a patente somente era interessante para o inovador quando este último não considerava possível preservar o seu segredo comercial.

Independentemente dessa crítica, o fato de que a opção por não patentear uma inovação, mantendo-a como segredo comercial, pode ser eficiente, tem sido reconhecida mesmo por autores mais próximos da teoria econômica ortodoxa. Mesmo autores como Friedman, Landes e Posner (1991), os quais acreditam que a opção pelo segredo comercial é frequentemente resultado de custos excessivos do sistema de patentes, ou de uma duração inadequada da proteção oferecida pela patente, admitem a escolha do segredo comercial.

Assim, é preciso reconhecer que a proteção de patentes não é essencial, de acordo com as evidências, para o incentivo à inovação em todos os setores da indústria. Provavelmente, as patentes são essenciais em um número limitado de setores, ainda que sejam setores importantes (como o farmacêutico).

Além dessa questão setorial, a defesa de uma proteção mais ampla e efetiva das patentes não é apresentada sem alguma controvérsia mesmo em livros-texto que apresentam a visão mais convencional (ver, por exemplo, Viscusi et al. (2000)). A razão disso é que patentes conferem monopólio sobre o emprego de uma tecnologia mais produtiva, ou a produção de um produto mais adequado às necessidades do consumidor.

Assim, uma patente acarreta um nível de produção menor do que aquele que seria alcançado na ausência deste instrumento legal, ao restringir a produção, limitando o acesso ao conhecimento que pode ser reproduzido a um custo marginal 
praticamente nulo e, deste modo, resulta em uma situação Pareto-ineficiente, se apenas nos limitarmos à visão ortodoxa mais óbvia.

$\mathrm{Na}$ verdade, mesmo nos Estados Unidos, hoje um dos países que lidera a defesa internacional por um maior rigor na proteção a patentes, até meados do século XX era o caráter monopolizador das patentes que era enfatizado, em detrimento de possíveis benefícios em termos de incentivos à inovação. Como explica Sell (2003, p. 66), até os anos oitenta do século XX, "Patent rights were construed as monopolies, market power was presumed and these rights were subordinated to the dominant anti-trust policy"2.

A ênfase sobre $o$ aspecto monopolizador das patentes somente se transferiu para $o$ incentivo que elas poderiam oferecer à invenção a partir dos anos oitenta, quando ficou evidente que “(...) while US firms pioneered technologies such as the transistor, the video cassette recorder, and the integrated circuit, other countries, most notably Japan, successfully commercialized these US inventions" (Sell, 2003, p. 67).

Há outras críticas, porém, que vão um pouco além da inadequação das patentes ao critério paretiano de eficiência competitiva. Boldrin e Levine (2002, p. 210) resumem algumas das críticas mais importantes às patentes, a maior parte delas enfatizando o papel das patentes na busca de rendas (rent seeking) e no emprego de práticas anticompetitivas.

É importante, assim, não esquecer que as patentes também são instrumento para a monopolização de mercados, tendo levantado preocupações nesse aspecto não apenas nos órgãos de defesa da concorrência em países desenvolvidos ${ }^{3}$, como também na pesquisa acadêmica ${ }^{4}$. São inúmeros os trabalhos que discutem e destacam a importância das patentes em práticas anticompetitivas ${ }^{5}$ e não há nenhuma razão para se acreditar que este tipo de utilização indevida de patentes será menor

\footnotetext{
${ }^{2}$ Do final do século XIX, com o aprovação do Sherman Act, até o final do século XX, "This anti-patent environment, characterized by vigorous anti-trust enforcement and judicial attacks on the scope and validity of patents, led US businesses to question the economic value of patent protection" (Sell, 2003. p. 66). Dada a ênfase na análise antitruste das patentes, "More often than not, the courts presumed patents to be invalid, and patentees were criticized for setting monopoly prices for inventions that were already in public domain". (Sell, 2003, p. 66).

${ }^{3}$ Ver, por exemplo, Marquis (2007) para o caso da União Europeia e Takigawa (2003) para o caso do Japão. Os Estados Unidos apresentam um debate tão intenso e volumoso sobre o problema das patentes (ao menos desde o caso do julgamento do pool de patentes de craqueamento de Petróleo em 1931), que é impossível citar aqui todos os trabalhos relevantes. A título de sugestão, uma breve síntese das preocupações norte-americanas mais comuns pode ser encontrada em Werner (1999).

${ }^{4} \mathrm{~A}$ preocupação com as possibilidades das patentes como instrumento de monopolização não é recente: ver Gilbert e Newbery (1982), Kamien e Schwartz (1982), Fudenberg, Gilbert e Stiglitz (1983) e Harris e Vickers (1985).

${ }^{5}$ Werner (1999), por exemplo, aponta algumas práticas competitivas derivadas da propriedade de patentes: cartéis (quando as patentes são licenciadas para competidores e utilizadas para fixar preços, reduzir quantidades ou dividir mercados), aumento do poder de mercado (especialmente quando o proprietário da patente adquire patentes em outras tecnologias competidoras), licenciamento de patente com cláusula de venda casada de outros produtos (tie in), licenciamento de patente com cláusula de proibição de uso de produtos ou de tecnologias de competidores (tie out) etc.
} 
no caso em que o proprietário da patente é uma empresa cuja sede se encontra em outro país ${ }^{6}$.

Os argumentos a favor das patentes se inspiram, na maior parte das vezes, em uma ideia de eficiência dinâmica, ou seja, de que sem o monopólio proporcionado pela patente às empresas inovadoras, não haveria incentivo adequado à inovação, e o ritmo do progresso técnico seria, em consequência, significativamente mais reduzido $^{7}$. Como explicam Braga, Fink e Sepulveda (2000, p. 27):

Intellectual creations have some characteristics of public goods. The blueprint for a new machine, the computer code for a software application, the script for a play, or a television broadcast can be simultaneously consumed by many agents at a zero (or at a very low) marginal cost. In short, the cost of reproduction of intellectual creation is typically a fraction of the cost of production. Although pricing at marginal cost would maximize consumer welfare from a static perspective, it would curtail incentives for investing in the creation of new intellectual works or improving existing knowledge. By granting temporary exclusive rights, IPRs are intended to allow property-holders to price their products above marginal cost and to recoup the initial knowledge-or information-generating investment ${ }^{8}$.

Obviamente esse argumento não pode ser aplicado diretamente quando se trata de justificar uma garantia de patente mais severa em países em desenvolvimento. De fato, uma vez que um país em desenvolvimento conceda uma proteção mais rigorosa para patentes de empresas em países desenvolvidos, isso pode significar apenas uma redução na produção do país em desenvolvimento (a ineficiência

\footnotetext{
${ }^{6}$ Até mesmo porque os sistemas de defesa da concorrência são mais frágeis em países em desenvolvimento. A possibilidade da utilização restritiva das patentes de empresas de países desenvolvidos em países em desenvolvimento já pode ser encontrado em Vaitsos (1972), que apontava para o fato de que as empresas de países desenvolvidos tendiam a solicitar patentes para para preservar mercados para seus produtos, impedindo a produção por parte de empresas locais.

${ }^{7}$ Essa justificativa das patentes é a mais comum, e a que produziu um maior número de trabalhos empíricos. Contudo, como mostram Mazzoleni e Nelson (1998, p. 1033), pode-se identificar ao menos três outras justificativas para a existência de patentes. Em uma justificativa alternativa, as patentes levariam os inovadores a revelarem (disclosure) as suas descobertas. Outra justificativa é a de que as patentes induzem os investimentos necessários para desenvolver e comercializar as inovações. Por último, as patentes tornariam possível uma exploração ordenada das perspectivas de inovação. Essas justificativas alternativas, com exceção da justificativa pelo incentivo à revelação da descoberta, se encontram muito pouco elaboradas, e não serão consideradas neste trabalho. A justificativa da revelação da descoberta está sujeita ao interesse de se patentear a descoberta, o que, conforme foi visto, pode não se verificar em determinados setores da indústria.

${ }^{8}$ Os mesmos autores fazem as ressalvas usuais quanto à solução representada pelas patentes, do ponto de vista da eficiência econômica, considerando-as uma solução segundo-melhor, dadas as características de bem público do conhecimento (Braga et al., 2000, p. 28).
} 
paretiana convencional), sem a contrapartida dos incentivos dinâmicos à inovação (um argumento que já se encontrava em Penrose (1973, p. 770)).

Assim, em uma situação em que a proteção rígida das patentes restringisse os ganhos de eficiência dinâmica às empresas em países desenvolvidos, enquanto aos países em desenvolvimento restasse apenas a ineficiência estática paretiana, teríamos a distribuição assimétrica entre perdas estáticas e ganhos dinâmicos, o que pode justificar uma abordagem mais flexível da proteção de patentes em países em desenvolvimento e não uma proteção mais rígida, como almejam os acordos internacionais e, mais recentemente, TRIPS.

Desse modo, a defesa da adesão a um padrão internacional único e mais rigoroso de proteção de patentes, por parte de um país em desenvolvimento, enfrenta várias dificuldades: a complexidade do tratamento das patentes, mesmo no âmbito da teoria econômica mais convencional; as evidências históricas de experiências bem-sucedidas de desenvolvimento, que privilegiaram opções adequadas às necessidades de cada país; os problemas anticompetitivos que o uso das patentes pode gerar, com efeitos negativos não apenas do ponto de vista estático, como também do ponto de vista dinâmico, para as indústrias dos países em desenvolvimento.

Resulta assim que a defesa da harmonização da proteção de patentes depende de forma essencial da possibilidade desta adesão promover a absorção de tecnologias mais avançadas, por parte das empresas do país em desenvolvimento, dada a assimetria internacional na produção de novas tecnologias, concentrada nos países desenvolvidos. Isso vincula diretamente a questão da proteção de patentes ao desenvolvimento econômico. Esse será o próximo assunto a ser abordado.

\section{PROTEÇÃO DE PATENTES E DESENVOLVIMENTO}

De acordo com o sentido de causalidade entre direitos de propriedade intelectual, especificamente patentes, e desenvolvimento econômico, os termos da discussão acerca da necessidade da proteção de direitos de propriedade intelectual são sensivelmente alterados. Assim, se a relação de causalidade se der no sentido de que uma maior proteção dos direitos de patente fornece um estímulo ampliado à invenção de novos produtos e processos, tem-se um argumento a favor do desenvolvimento de um sistema de proteção de patentes rigoroso como precondição do crescimento e do desenvolvimento.

Já se o contrário for verdade, isto é, se o desenvolvimento econômico é que incentiva à proteção das patentes, a situação pode se inverter, e os argumentos a favor da proteção mais severa de patentes podem resultar muito enfraquecidos. Isso, especialmente se for verificado na prática que os países passam a defender uma proteção mais rigorosa de patentes somente após as empresas nacionais adquirirem capacitação tecnológica em nível internacional, tendo estas empresas anteriormente se beneficiado de um regime pouco severo, ou mesmo inexistente de proteção de patentes.

O tipo de relação de causalidade em que a proteção dos direitos de propriedade 
sobre patentes favorece o desenvolvimento econômico, ao estimular a atividade inventiva e o incremento de novos produtos e processos na economia, é explicitamente assumido por autores como o Prêmio Nobel Douglass C. North (North, 1990, p. 75):

The development of an incentive structure through patent laws, trade secret laws, and other laws raised the rate of return on innovation and also led to the development of the invention industry and its integration into the way economies evolved in the Western world in modern times, which in turn underlay the Second Economic Revolution?.

Outros autores, contudo, demonstram que a relação entre proteção de patentes e desenvolvimento parece ser bem menos direta e linear do que a leitura do trecho acima poderia a princípio sugerir. Esse é o caso, por exemplo, de Keith E. Maskus (2000). Com efeito, considerando a literatura acerca das evidências empíricas, Maskus (2000, p. 144) verifica que parece haver uma relação não-linear entre o aumento da proteção às patentes e o desenvolvimento econômico: à medida que aumenta a renda per capita de um país, o grau de proteção de patentes se reduz, voltando a aumentar apenas para níveis de renda per capita elevados.

Essa relação não-linear daria origem a uma curva de proteção de direitos de propriedade intelectual e patentes em "U", ou seja, a proteção por patentes diminui à medida que o grau de desenvolvimento atinge níveis intermediários, para tornar a aumentar quando níveis elevados de desenvolvimento são atingidos ${ }^{10}$. A razão para isso estaria nas mudanças no padrão de geração e incorporação de tecnologia, ao longo das diferentes fases do desenvolvimento econômico:

It therefore seems that as incomes and technical capabilities grow to intermediate levels, adaptive innovation emerges, but competition remains focused largely on imitation, so that the bulk of economic and political interests prefer weak protection. As economies mature to higher levels of technological capacity and as demands for high-quality, differentiated products increase, more domestic firms favor effective IPRs. Finally, at the highest income levels the strength of IPRs shifts up sharply (...) (Maskus, 2000, p. 144).

Falvey e Greenaway (2006) encontraram evidências favoráveis a uma curva em U relacionando proteção de direitos de propriedade intelectual e crescimento

\footnotetext{
${ }^{9}$ Curiosamente, ao mesmo tempo em que utiliza o caso das patentes para ilustrar como direitos de propriedade estimulam o investimento privado, North $(1981,2005)$ também aponta a Holanda como exemplo de sucesso na Europa, pela proteção que conferiu aos direitos de privados propriedade. Contudo, a Holanda foi o último país a adotar uma legislação de patentes na Europa, em 1912. Também a Suíça reduziu expressivamente o nível de proteção oferecido às patentes em 1850 , e somente voltou a adotar efetivamente a proteção de patentes em 1907 (La Croix e Konan, 2006, p. 5).

${ }^{10}$ Para uma discussão da curva de proteção de patentes em função do nível de desenvolvimento econômico com a forma de "U", veja-se Evenson e Westphal (1997).
} 
econômico. Ao analisarem um painel com 79 países e empregando técnica econométrica capaz de identificar thresholds, Falvey e Greenaway (2006) encontraram evidências de que países com renda per capita elevada tendem a crescer mais rapidamente quanto maior a proteção de direitos de propriedade intelectual.

Embora esse resultado não seja surpreendente, uma vez que são esses países os que concentram o maior volume de investimento em P\&D, Falvey e Greenaway (2006) identificaram relações assimétricas entre a proteção de propriedade intelectual e o crescimento dos países de renda per capita mais baixa: enquanto que a proteção pode ter impacto positivo significativo no crescimento dos países mais pobres, nenhuma relação significativa no caso dos países de renda intermediária foi encontrada (Falvey e Greenaway, 2006, p. 712).

Com efeito, a hipótese de uma curva de proteção de direitos de propriedade intelectual e patentes em " $U$ " somente pode ser explicada a partir do argumento de que a aceleração do desenvolvimento, quando este se encontra em um patamar intermediário, exige o afrouxamento da proteção de patentes. Resulta difícil conciliar este resultado com a ideia de uma relação simples e direta entre proteção de patentes e promoção de desenvolvimento.

É importante notar que a hipótese de uma curva de proteção de patentes em "U” é uma hipótese que tem encontrado sustentação empírica não apenas em análises cross-section, mas também em vários exemplos históricos: países atualmente desenvolvidos, quando se encontravam em níveis intermediários de desenvolvimento, relaxaram a proteção de algum direito de propriedade intelectual. No caso norte-americano, ao mesmo tempo em que a legislação de patentes no século XIX ganhava um desenvolvimento expressivo, os direitos de reprodução e cópia (copyrights) foram ignorados ao longo do século XIX nos Estados Unidos, não obstante os protestos internacionais, especialmente da Grã-Bretanha. Grande consumidor de literatura produzida no exterior, os Estados Unidos ignoraram os apelos pelo respeito aos copyrights até 1891 (La Croix e Konan, 2006).

Não apenas no caso norte-americano o sistema de proteção de direitos de propriedade intelectual foi desenhado de forma a atender aos interesses de desenvolvimento daquele país. Da mesma forma que outras nações da Europa, a Grã-Bretanha ao longo do século XIX não concedia patente a não-residentes e, caso fosse concedida uma patente em relação a uma inovação gerada em país estrangeiro, esta patente era acompanhada de um working requirement, ou seja, da obrigação de que a patente fosse aplicada em atividade produtiva, para permanecer em vigência ${ }^{11}$.

Assim, historicamente a proteção de direitos de propriedade foi sempre definida de acordo com os interesses do desenvolvimento dos países, de acordo com suas necessidades de desenvolvimento. Essa situação mudou significativamente após TRIPS, como será visto a seguir.

\footnotetext{
${ }^{11}$ Ver, a esse respeito, Khan e Sokoloff (2001). As duas práticas - a não concessão de patentes a estrangeiros e a imposição de working requirements são proibidas pelo acordo TRIPS.
} 


\section{O ACORDO TRIPS E A HARMONIZAÇÃO DE PATENTES}

Verifica-se, a partir das duas últimas décadas, uma tendência crescente de associação de acordos comerciais internacionais com direitos de propriedade intelectual, cuja expressão mais destacada é o acordo TRIPS, o qual resultou da conclusão da Rodada Uruguai em 1994 em Marrakech. O acordo TRIPS abrange diversos aspectos dos direitos de propriedade intelectual: patentes, marcas, direitos autorais, indicação geográfica, desenho industrial, desenho de layout de circuitos integrados etc.

O fato a ser destacado, contudo, é a desconsideração no acordo TRIPS das diferenças entre os países, no que diz respeito aos seus diferentes graus de desenvolvimento e à necessidade de tratamento diferenciado. Note-se que o reconhecimento da necessidade de um tratamento diferenciado e mais favorável aos países menos desenvolvidos foi um princípio fundamental do Acordo Geral de Tarifas e Comércio (GATT), princípio esse que foi estendido para a Organização Mundial do Comércio (WTO/OMC).

Todavia, o mesmo princípio não foi estendido a TRIPS: TRIPS não possui diferenças significativas nas regras que são aplicadas aos países em desenvolvimento, em relação às regras dos países desenvolvidos. As provisões de tratamento especial e diferenciado em TRIPS, sinteticamente, se resumem a:

1. Períodos de transição para a maioria dos aspectos do acordo (artigos 65, seção 2 , e 65 , seção 4), com períodos maiores para os países menos desenvolvidos (seis anos extras) e menores para os países em desenvolvimento (quatro anos extras). Cinco anos adicionais foram concedidos para a introdução de patentes em setores que anteriormente não eram adequadamente protegidos.

2. Assistência técnica e financeira para facilitar a implantação do acordo, em termos mutuamente acordados entre países desenvolvidos e não desenvolvidos (artigo 67).

3. Transferência de tecnologia a ser encorajada para os países menos desenvolvidos (e não a todos os países não-desenvolvidos), pelo incentivo nos países desenvolvidos a instituições e empresas com este objetivo (artigo 66 ,seção 2). Aqui é importante diferenciar o artigo 7 do artigo 66 e sua seção 2. O artigo 7 possui caráter genérico e afirma que a proteção de PI deve contribuir para a difusão e disseminação da tecnologia para a vantagem mútua. $\mathrm{O}$ artigo 66 , seção 2, menciona incentivos a empresas e instituições de países desenvolvidos que promovam transferência de tecnologia, especificamente para os países menos desenvolvidos (embora também não contenha nenhuma determinação mais efetiva).

Pode-se afirmar, assim, que em relação aos acordos econômicos multilaterais, TRIPS é fortemente assimétrico, na medida em que adota uma abordagem one fits 
all. Com efeito, a ideia de um modelo único não é isenta de controvérsias. $\mathrm{Na}$ verdade, a ideia de que há somente um único padrão de proteção de direitos de propriedade intelectual adequado pode ser questionada, mesmo no âmbito de uma abordagem teórica mais convencional. Assim, mesmo Keith E. Maskus, um autor mainstream comprometido com a defesa de sistemas de proteção de propriedade intelectual, reconhece que estes sistemas podem variar em seu desenho, de acordo com as necessidades de cada país.

Ao analisar o sistema de patentes adotado pelo Japão no início do século XX, Maskus (2000, p. 143, grifo do original) observa que:

It was designed to encourage industrial development through emphasizing technology acquisition from abroad, domestic information diffusion, and incremental innovation. In short, the system was developed with the interests of a technology follower in mind. The Japanese regime significantly limited patent scope and breadth. For example, pharmaceutical patents were not provided until the 1970s.

Coerentemente, Maskus (2000, p. 144) reconhece que "The terms on which a country might wish to protect IPRs [intellectual property rights] depend on, among other things, its position on the global technology ladder and on social concerns". Essa visão que reconhece as diferenças nos processos de desenvolvimento dos países, contudo, foi excluída do horizonte de acordos internacionais tais como TRIPS, que busca uniformidade na proteção de direitos de propriedade intelectual, independentemente do grau de desenvolvimento atingido pelos países.

Vale a pena notar que TRIPS uniformizou a duração mínima das patentes em 20 anos, superando os 17 anos que eram norma nos EUA no momento da adoção de TRIPS, e não permite aos governos excluir setores industriais das patentes (exceto certa flexibilidade para variedades de plantas). O ponto importante a ser percebido aqui é a perda significativa de flexibilidade do emprego de PI como instrumento de política de desenvolvimento. Isso, juntamente com uma proteção mais severa de PI, pode gerar significativas transferências de renda dos países em desenvolvimento para os países desenvolvidos.

Os países em desenvolvimento podem reduzir alguns dos custos do sistema de patentes via licenciamento compulsório (artigo 31). Contudo, as circunstâncias para isso são bem definidas e limitadas: emergência nacional, ou extrema urgência, com uso público não comercial. Além disso, como a própria declaração de Doha sobre o Acordo TRIPS e saúde pública reconhece, essa flexibilização, para se tornar realidade, pode exigir que o país não desenvolvido tenha capacidade de produção interna. Assim, essa flexibilização do acordo TRIPS possui na prática um alcance ainda mais limitado do que pode parecer a princípio.

Há outros pontos do acordo TRIPS que envolvem mudanças radicais em relação à situação anterior, reduzindo significativamente os graus de liberdade dos países em desenvolvimento. Além de proibir o tratamento discriminatório entre 
nações e com relação a cidadãos estrangeiros (o que exclui a possibilidade de aplicação de regras envolvendo working requirement), produtos e processos agora são obrigatoriamente patenteáveis ${ }^{12}$.

Porém, apesar dessa redução significativa na liberdade dos países em desenvolvimento, um argumento ainda pode ser levantado em defesa de uma proteção mais severa das patentes nos países em desenvolvimento. Esse argumento diz respeito às formas de difusão internacional de tecnologia, ou seja, pode-se indagar se uma proteção mais severa de patentes incentivaria a difusão internacional de tecnologia. Em caso afirmativo, ainda que sejam consideradas as ressalvas apresentados anteriormente, novamente haveria um ponto importante a favor de proteção mais rígida das patentes como instrumento de desenvolvimento.

Ocorre que se a difusão acontece por meio do comércio internacional, mais particularmente da importação de bens que embutem tecnologia de ponta, então o reconhecimento das patentes se torna menos importante. Caso a difusão de tecnologia ocorra pelo comércio internacional, a questão passa a ser liberalizar ou não os fluxos comerciais, e não a proteção das patentes.

Contudo, se a principal forma de difusão de tecnologia é o investimento direto estrangeiro (IDE), então passa a fazer sentido argumentar a favor de uma proteção mais significativa das patentes, pois se pode sustentar (ao menos em tese) que o IDE portador de tecnologia mais sofisticada não irá se dirigir para países que não garantem as suas patentes ${ }^{13}$. Esse será o próximo tema a ser abordado.

\section{DIFUSÃO INTERNACIONAL DE TECNOLOGIA E DESENVOLVIMENTO}

De uma forma geral, há três formas de difusão de tecnologias: comércio internacional de bens intermediários, divulgação internacional dos resultados de pesquisa e desenvolvimento (P\&D) e investimento direto estrangeiro (ver, a esse respeito, Keller (2004)). A literatura não considera, contudo, que esses mecanismos possuem a mesma capacidade para a difusão internacional de tecnologia.

Com efeito, o comércio internacional de produtos intermediários pode ser considerado uma fonte fraca de difusão internacional de tecnologia, uma vez que, com o emprego de produtos intermediários importados, não é a tecnologia que é

\footnotetext{
$12 \mathrm{O}$ reconhecimento de patentes de produtos e processos foi usado discricionariamente, mesmo em períodos mais recentes e por países desenvolvidos, de acordo com as necessidades de sua indústria. Por exemplo, até 1967 na antiga República Federal da Alemanha, 1979 na Itália e 1992 na Espanha, não eram reconhecidas as patentes de produtos farmacêuticos (cf. Chang, 2001, p. 7).

${ }^{13}$ Maskus (2000, p. 147) coloca a questão da defesa do papel dos direitos de propriedade intelectual no desenvolvimento claramente nesses termos, ao discutir as limitações que as defesas usuais da proteção de direitos de propriedade em geral enfrentam quando se trata de direitos de propriedade intelectual.
} 
diretamente incorporada, mas apenas os produtos que resultam dela (Keller, 2004, p. 755). Isso pode ser uma limitação ainda mais severa quanto maior o volume de conhecimento tácito ${ }^{14}$ envolvido na produção do bem intermediário ${ }^{15}$.

No que diz respeito à avaliação das evidências empíricas acerca dos efeitos do comércio internacional na difusão de tecnologia, iniciada a partir de Coe e Helpman (1995), os resultados são, em grande medida, convergentes quanto à importância do comércio internacional para a difusão tecnológica. Contudo, os trabalhos que apresentam esses resultados têm sofrido críticas metodológicas bastante severas, que põem em xeque, em maior ou menor grau, esses resultados ${ }^{16}$.

Todavia, é importante observar que a grande maioria dos estudos empíricos que procuram avaliar a difusão tecnológica proporcionada pelo comércio internacional não servem para distinguir a importância relativa do IDE e do comércio. Isso porque a importação de bens de capital de elevado conteúdo tecnológico frequentemente ocorre em função de investimentos de empresas multinacionais, de modo que é preciso distinguir o efeito positivo do comércio internacional na difusão tecnológica da atividade de empresas multinacionais.

Lee (2006) é um dos (poucos) autores a fazer a distinção entre o efeito do comércio internacional e do influxo de IDE para a difusão de tecnologia. Utilizando um painel de 16 países da OCDE, Lee (2006) apresenta evidências de que a difusão de conhecimento produzida pelo influxo de IDE e pelos canais de difusão que não estão relacionados a transações comerciais (bancos internacionais de patentes, publicações técnicas, etc.) são significantes e robustos. $\mathrm{O}$ mesmo não acontece com o investimento realizado pelas empresas nacionais no exterior e as importações de bens intermediários ${ }^{17}$.

Resulta então que o IDE pareceria ser o principal canal de difusão internacional de tecnologia. Isso, por dois motivos: (a) tecnologia específica com relação à firma é transferida através das fronteiras internacionais, em função do compartilhamento de tecnologia entre a matriz e suas subsidiárias; (b) haveria a difusão tecnológica das subsidiárias multinacionais para as empresas locais, via rotatividade de mão-de-obra e demanda por insumos de maior qualidade (para uma síntese da literatura, cf. Keller, 2004).

Para discutir os efeitos das patentes sobre o IDE é preciso analisar as evidências de que uma proteção mais forte de patentes (e de direitos de propriedade in-

\footnotetext{
${ }^{14}$ Muito resumidamente, conhecimento tácito é aquele que não é passível de codificação científica (cf. Polanyi (1974)).

${ }^{15} \mathrm{O}$ papel das exportações como instrumento de difusão internacional de tecnologia para o país exportador é muito mais controverso. Para duas evidências antagônicas quanto ao papel das exportações como instrumento de difusão internacional de tecnologia, cf. Hallward-Driemeier, Iarossi e Sokoloff (2002) e Clerides, Lach e Tybout (1998).

${ }^{16}$ Para uma resenha crítica desses resultados, ver Cincera e Van Pottelsberghe de La Potterie (2001).

${ }^{17}$ Hejazi e Safarian (1999) obtiveram resultados semelhantes, em que o impacto do IDE se mostrou mais importante que o do comércio internacional.
} 
telectual em geral) é capaz de incentivar o ingresso do IDE. Isso, uma vez que se argumenta que, sem proteção severa de patentes, o IDE não ingressaria em países em desenvolvimento, pois os direitos de propriedade sobre as tecnologias e produtos não estariam garantidos.

Em relação aos fatores que atrairiam o IDE, o que inclui a proteção de direitos de propriedade e patentes em particular, tem sido verificado empiricamente que as dimensões dos mercados consumidores nacionais são um fator importante na atração de IDE. Esta relação foi verificada por Zejan (1990), que encontrou uma correlação significativa entre o investimento em P\&D das empresas suecas e o PIB dos países hóspedes. Kumar (1996) confirmou o resultado de que países com grandes mercados são mais atraentes para investimento em P\&D por parte das empresas multinacionais.

Outros dois fatores que parecem ter influência significativa são a qualificação da mão-de-obra no país hospedeiro e a possibilidade da empresa multinacional se beneficiar de spillovers locais (cf. Blömstrom e Kokko (2003) e Sanyal (2004)). Contudo, no que diz respeito à atratividade fornecida por uma proteção mais ampla e severa de patentes (e direitos de propriedade em geral), as evidências são frágeis.

Lee e Mansfield (1996) encontraram uma correlação positiva entre a percepção de executivos de grandes empresas norte-americanas quanto ao nível de proteção de direitos de propriedade intelectual em países em desenvolvimento e seu efeito sobre o nível de investimento destas empresas nestes países. Contudo, a percepção de direitos de propriedade frágeis pode na verdade sintetizar uma avaliação geral de que o país em questão não possui uma ambiente institucional adequado ao IDE, e como os executivos não foram consultados sobre os demais fatores institucionais destes países, não é possível afirmar que foi especificamente a má proteção de propriedade intelectual o problema.

Branstetter et al. (2007) procuram fornecer evidências empíricas quanto ao aumento da transferência de tecnologia por parte de empresas multinacionais após reformas em países em desenvolvimento que tenham aumentado a proteção de direitos de propriedade intelectual. Contudo, como Branstetter et al. (2007) empregam os pagamentos de royalties como indicador de transferência de tecnologia, há a forte possibilidade de que na verdade tenham captado não um aumento na transferência de tecnologia, mas sim um aumento na extração de royalties possibilitado pela proteção mais rigorosa de propriedade intelectual.

Além dessas críticas, também há uma série de evidências contrárias à tese de que a proteção de direitos de propriedade intelectual é precondição para atrair IDE. Kumar (1996) verificou que a atratividade oferecida pela garantia dos direitos de propriedade intelectual somente se mostrou significativa para o caso de países desenvolvidos. Maskus (1998), apesar de explicitamente procurar defender a ideia de que proteção de direitos de propriedade intelectual mais rígida atrai IDE, obtém uma correlação negativa entre o volume de pedidos de patentes de empresas multinacionais e o grau de proteção de patentes em países em desenvolvimento. 
Também Sanyal (2004) tentou estimar o impacto que a segurança dos direitos de propriedade intelectual tem sobre os gastos com P\&D de empresas multinacionais norte-americanas. Sanyal (2004) verificou que a proteção de patentes apresentou coeficiente negativo quando foram considerados apenas países em desenvolvimento ${ }^{18}$. Analisando os resultados obtidos em uma síntese que resume os aspectos mais importantes identificados, Sanyal (2004, p. 69) observou que:

(...) R\&D based in developing economies are geared more towards adaptations and serving local or regional markets. These adaptations may not be affected by the availability of patent protection. The only factor that affects the decision to invest in $R \& D$ facilities in developing economies is US sales.

\section{CONCLUSÕES}

Não se verifica, na literatura econômica, resultados que justifiquem a adoção de um padrão internacional uniforme e mais rigoroso de proteção de patentes. Pelo contrário, tanto a teoria econômica, as evidências históricas do tratamento das patentes por parte dos países desenvolvidos, a presença de uma curva em U relacionando proteção de direitos de propriedade intelectual e nível de desenvolvimento, e a aparente ausência de um papel significativo das patentes e dos direitos de propriedade intelectual na atração de IDE colocam em xeque a tendência à harmonização da proteção de patentes consagrada no acordo TRIPS.

$\mathrm{Na}$ verdade, podem ser encontrados fortes elementos para dar sustentação à tese contrária: há sem dúvida motivos para se acreditar ser fundamental cada país em desenvolvimento adequar seu regime de proteção de patentes as suas necessidades.

O acordo TRIPS busca aumentar significativamente o patamar de proteção aos direitos de propriedade intelectual (patentes, marcas e direitos autorais), o que, no caso dos países em desenvolvimento, significa limitar a capacidade das empresas nacionais se apropriarem e desenvolverem tecnologias empregadas em países desenvolvidos sem incorrerem nos custos resultantes da proteção de direitos de propriedade intelectual, em especial as patentes.

As evidências apontadas neste trabalho sugerem que a possibilidade de se buscar uma maior flexibilidade na tendência de harmonização que embasa o acordo TRIPS, identificada por Michalopoulos (2003), é necessária para o desenvolvimento econômico de países em desenvolvimento.

\footnotetext{
${ }^{18}$ Pfister e Deffains (2005) analisaram a importância da garantia dos direitos de patentes para as empresas francesas em 17 países em desenvolvimento, não encontrando relação significativa.
} 


\section{REFERÊNCIAS BIBLIOGRÁFICAS}

BESSEN, James. (2005) "Patents and the diffusion of technical information." Economic Letters, 86 (1): 121-128.

BLÖMSTROM, Magnus; KOKKO, Ari. (2003) “The Economics of Foreign Direct Investment Incentives." Cambridge, Mass.: NBER Working Paper 9489.

BOLDRIN, Michele; LEVINE, David. (2002) "The Case Against Intellectual Property". The American Economic Review, 92 (2): 209-212.

BRAGA, Carlos A. Primo; FINK, Carsten; SEPULVEDA, Claudia Paz. (2000) "Intellectual Property Rights and Economic Development”. Washington: World Bank, World Bank Discussion Paper n. 412.

BRANSTETTER, Lee; FISMAN, Raymond; FOLEY, C. Fritz; SAGGI, Kamal. (2007) "Intellectual Property Rights, Imitation, And Foreign Direct Investment: Theory and Evidence." Cambridge, Mass.: NBER Working Paper 13033.

CHANG, Ha-Joon. (2001) “Technology Transfer, Intellectual Property Rights, and Industrial Development in Developing Countries: a Background Paper Prepared for World Industrial Development Report, 2001 of the UNIDO (second draft). Vienna: UNIDO.

CINCERA, Michele; VAN POTTELSBERGHE DE LA POTTERIE, Bruno. (2001) "International R\&D Spillovers: A Survey.” Brussels Economic Review, 44 (1): 3-32.

CLERIDES, Sofronis; LACH, Saul; TYBOUT, James. (1998) "Is Learning by Exporting Important? Micro-Dynamic Evidence from Colombia, Mexico, and Morocco". The Quarterly Journal of Economics, 113 (3): 903-947.

COE, David T.; Helpman, Elhanan. (1995) “International R\&D Spillovers.” European Economic Review, 39 (5): 859-887.

COHEN, Wesley M.; NELSON, Richard R.; WALSH, John P. (2000) "Protecting Their Intellectual Assets: Appropriability Conditions and Why U.S. Manufacturing Firms Patent (or Not). Cambridge, Mass.: NBER Working Paper 7552.

EVENSON, Robert E.; WESTPHAL, Larry E. (1997) “Technological Change and Technology Strategy". In CHENERY, Hollis; SRINIVASAN, T. N., Handbook of Development Economics, vol. 3, Amsterdam: North-Holland.

FALVEY, Rod, Foster Neil; GREENAWAY, David. (2006) "Intellectual Property Rights and Economic Growth". Review of Development Economics, 10 (4): 700-719.

FRIEDMAN, David D.; LANDES, William M.; POSNER, Richard A. (1991) "Some Economics of Trade Secret Law". The Journal of Economic Perspectives, 5 (1): 61-72.

FUDENBERG, Drew; GILBERT, Richard; STIGLITZ, Joseph E. (1983) "Preemption, Leap-frogging, and Competition in Patent Races." European Economic Review, 22 (1): 3-31.

GILBERT, Richard J; NEWBERY, David M. G. (1982) "Preemptive Patenting and the Persistence of Monopoly". The American Economic Review, 72(3): 514-526.

HALLWARD-DRIEMEIER, Mary; IAROSSI, Guiseppe e SOKOLOFF, Kenneth. (2002) "Exports and Manufacturing Productivity in East Asia: A Comparative Analysis with Firm-Level Data". Cambridge, Mass.: National Bureau of Economic Research Working Paper n. 8894.

HARRIS, Christopher; VICKERS, John. (1985) "Patent Races and the Persistence of Monopoly," in GEROSKI, Paul A.; PHLIPS, Louis; ULPH, Alistair (eds.), Oligopoly, Competition, and Welfare. New York: Basil Blackwell.

HEJAZI, Walid; SAFARIAN, A. Edward. (1999) “Trade, Foreign Direct Investment, and R\&D Spillovers.” Journal of International Business Studies, 30 (3): 491-511.

KAMIEN, Morton I.; SCHWARTZ, Nancy L. (1982) Market Structure and Innovation. Cambridge: Cambridge University Press.

KHAN, B. Zorina; SOKOLOFF, Kenneth (2001). "History Lessons: The Early Development of Intellectual Property Institutions in the United States". The Journal of Economic Perspectives, 15 (3): 233-246.

KELLER, Wolfgang. "International Technology Diffusion". (2004) Journal of Economic Literature, XLII (3): 752-782. 
KUMAR, Nagesh. (1996) "Intellectual Property Protection, Market Orientation and Location of Overseas R\&D Activities by Multinational Enterprises.” World Development, 24 (4): 673-688.

LA CROIX, Sumner; KONAN, Denise Eby. (2006) "Have Developing Countries Gained From the Marriage Between Trade Agreements and Intellectual Property Rights?” Honolulu: UH-Manoa Economics Working Paper $n^{\circ} 06-5$.

LEE, Gwanghoon. (2006) “The Effectiveness of International Knowledge Spillovers Channels.” European Economic Review, 50 (8): 2075-2088.

LEE, Jeong-Yeon; MANSFIELD, Edwin. (1996) "Intellectual Property Protection and U.S. Foreign Direct Investment". The Review of Economics and Statistics, 78 (2): 181-186.

LEVIN, Richard C.; KLEVORIC, Alvin K.; NELSON, Richard R.; WINTER, Sidney G. (1987). "Appropriating the Returns Form Industrial Research and Development". Brookings Papers on Economic Activity - Microeconomics, 3: 783-818.

MACHLUP, Fritz; PENROSE, Edith. (1950) "The Patent Controversy in the Nineteenth Century. The Journal of Economic History. 10 (1): 1-29.

MANSFIELD, Edwin. (1986) "Patents and Innovation: An Empirical Study". Management Science, 32 (2): 173-81.

MANSFIELD, Edwin; SCHWARTZ, Mark; WAGNER, Samuel. (1981) "Imitation Costs and Patents: An Empirical Study”. The Economic Journal, 91 (364): 907-918.

MARQUIS, Mel. (2007) "Patent, Know-How, and Software Copyright Licensing: The EU Competition Regime". Antitrust Bulletin, 52 (2): 239-331.

MASKUS, Keith E. (2000) Intellectual Property Rights in the Global Economy. Washington, DC: Institute for International Economics.

. (1998) "The International Regulation of Intellectual Property." Weltwirtschaftliches Archiv, 134 (2): 186-208.

MAZZOLENI, R.; NELSON, R. N. (1998) “The Benefits and Costs of Stronger Patent Protection: A Contribution to the Current Debate”. Research Policy, 27 (3): 273-84.

MICHALOPOULOS, Constantine. (2003) "Special and Differential Treatment of Developing Countries in TRIPS". Geneva: QUNO. Disponível em http://www.qiap.ca/documents/DT(US)1.pdf

NORTH, Douglass C. (1981) Structure and Change in Economic History. New York: W. W. Norton \& Company.

. (1990) Institutions, Institutional Change and Economic Performance. Cambridge: Cambridge University Press.

(2005) Understanding the Process of Economic Change. Princeton: Princeton University Press.

ORDOVER, Janusz A. (1991) “A patent system for both diffusion and exclusion”. Journal of Economic Perspectives, 5 (1): 43-60.

PENROSE, Edith. "International Patenting and the Less Developed Countries." (1973) The Economic Journal, 83 (331): 768-786.

PFISTER, Etienne; DEFFAINS, Bruno. (2005) "Patent Protection, Strategic FDI and Location Choices: Empirical Evidence from French Subsidiaries' Location Choices in Emerging Economies." International Journal of the Economics of Business, 12 (3): 329-346.

POLANYI, Michael. (1974) Personal Knowledge: Towards a Post-Critical Philosophy. Chicago: Chicago University Press.

SANYAL, Prabuddha. (2004) "Intellectual property rights protection and location of R\&D by multinational enterprises." Journal of Intellectual Capital, 5 (1): 59-76.

SCHERER, Frederic M. (1984) Innovation and Growth. Cambridge, Mass.: The MIT Press. ; ROSS, D. (1990). Industrial Market Structure and Economic Performance. Boston: Houghton Mifflin Co.

SELL, Susan K. (2003) Private Power, Public Law: The Globalization of Intellectual Property Rights. Cambridge: Cambridge University Press.

TAKIGAWA, Toshiaki. (2003) "Antitrust Intervention in Intellectual Property Licensing and Unilateral Refusal to License: a comparative analysis of Japanese and US Policies.” Antitrust Bulletin, 48 (4): 885-921.

VAITSOS, Constantine V. (1972) "Patents Revisited: Their Function in Developing Countries". Journal of Development Studies, 9 (1): 71-97. 
VISCUSI, W. Kip; VERNON, John M.; HARRINGTON JR., Joseph E. Economics of Regulation and Antitrust. Cambridge, Mass: The MIT Press, 2000.

WERNER, Jacques. (1999) “Antitrust and Intellectual Property: The Uneasy Relationship.” The Journal of World Intellectual Property, 2 (3): 417-422.

ZEJAN, Mario C. (1990) "R \& D Activities in Affiliates of Swedish Multinational Enterprises." The Scandinavian Journal of Economics, 92 (3): 487-500. 